\title{
Grain-Boundary Enhanced Diffusion from Instantaneous Source in Small-Grain Deep-Penetration Condition
}

\author{
Atsuomi FUKUURA and Yasumichi OISHI ${ }^{\dagger}$ \\ Central Research Laboratory, Kyocera Corporation, Kokubu-shi, Kagoshima 899-43 \\ 小粒径深侵入条件における薄膜源からの粒界拡散 \\ 福浦篤臣・大石行理 ${ }^{\dagger}$ \\ 京セラ(株)総合研究所, 899-43 鹿児島県国分市山下町 1-4
}

[Received December 17, 1993; Accepted July 21, 1994]

\begin{abstract}
A grain-boundary diffusion equation was derived for small-grain deep-penetration diffusion and solved for the instantaneous source condition, where the flux from the grain boundary into grains was taken into account as a time-dependent function. From the solution of the equation, the average concentration distribution along the diffusion direction was derived to describe the concentration distribution to be determined for polycrystal by scanning technique with electron probe X-ray microanalyzer (EPMA).
\end{abstract}

Key-words : Grain-boundary diffusion, Small-grain deeppenetration condition, Instantaneous source, Polycrystal, Concentration distribution

\section{Introduction}

Quantitative analysis of grain-boundary enhanced diffusion was first conducted by Fisher in 1951.1) The physical model in his analysis is described by a crystal bulk of low diffusivity bisected perpendicularly to the plane surface by a thin slab, that is grain boundary, of high diffusivity with a constant diffusant concentration kept on the surface. His equation allows calculation of the concentration for given bulk and grain-boundary diffusion coefficients as a function of time, diffusion depth along the grain boundary, and the distance from the grain boundary to the crystal interior. Fisher's analysis assumes a stationary concentration distribution along the grain boundary by ignoring the effect of the diffusion flux from the grain boundary to the bulk. Consequently, application of Fisher's equation yields accurate results only when the ratio of grain-boundary to bulk diffusion coefficient is sufficiently large and diffusion time is relatively long.2) However, as the ratio decreases below a certain magnitude, the error tends to become higher.

Whipple ${ }^{3)}$ derived, for the same physical model

\footnotetext{
+ Now with Institute for Study of the Earth's Interior, Okayama University, Misasa-cho, Tohaku-gun, Tottori 682-01 現在 : 岡山大学地球内部研究センター, 682-01 鳥取県東伯 郡三朝町
}

with a constant source as Fisher's model, his grainboundary diffusion equation by taking account of the diffusion flux from the grain boundary to the bulk in describing the concentration distribution in the grain boundary.

Suzuoka ${ }^{4}$ later derived his equation for the same physical model but with the instantaneous source condition. Suzuoka's equation is useful for the analysis of the case where thin film of a tracer is deposited on the surface of the sample.

Those grain-boundary diffusion equations by Fisher, Whipple and Suzuoka were all derived for the boundary condition where the size of the crystal bulk is large, the diffusion time relatively short and consequently, the diffusion penetration relatively short, as may be called "large-grain short-penetration" diffusion. In contrast there are many cases of "smallgrain deep-penetration" diffusion, as in sintered ceramics where diffusion proceeds over the distance beyond the size of crystal grains. For this small-grain deep-penetration diffusion, Oishi and Ichimura ${ }^{5)}$ derived a grain-boundary diffusion equation for the constant surface concentration with the assumption of stationary concentration distribution in the grain boundary. This assumption enabled them to determine simultaneously the parameters of bulk and grain-boundary diffusion from the logarithmic concentration distribution plotted against diffusion distance. This convenient technique has been applied to analyses of small-grain deep-penetration diffusion in fluorite-type cubic, ${ }^{5)-11)}$ tetragonal, ${ }^{12}$ and C-type cubic $^{13)}$ polycrystalline $\mathrm{ZrO}_{2}$-base solid solutions. Application of the "large-grain short-penetration" diffusion equations to such "small-grain deep-penetration" diffusion tends to result in overestimated grainboundary diffusion coefficients. ${ }^{10)}$ However, the grain-boundary diffusion parameters determined in the large-grain short-penetration condition using Fisher's and Whipple's equations agreed with that determined in the small-grain deep-penetration condition using the Oishi and Ichimura equation for cationic interdiffusion in a C-type cubic $\mathrm{Y}_{2} \mathrm{O}_{3}-\mathrm{ZrO}_{2}-$ $\mathrm{HfO}_{2}$ solid solution. ${ }^{13)}$ Sound application of the Oishi and Ichimura equation should be limited to the cases 
of relatively large ratio of the grain-boundary to the bulk diffusion coefficient and relatively long diffusion time for the assumption of stationary concentration distribution in the grain boundary.

The purpose of the present work is to derive a grain-boundary diffusion equation for the small-grain deep-penetration diffusion from the instantaneous source, where the diffusion flux from the grain boundary to the bulk is to be taken into account in calculating the grain-boundary concentration.

\section{Derivation of the grain-boundary diffusion equation}

The present small-grain deep-penetration diffusion is schematically described by Fig. 1 . Here the polycrystal consisting of equal-sized grains is in contact with the thin layer, which is the instantaneous source of the diffusant. The diffusion coefficient in the grain boundary, $D^{\prime}$, is assumed to be greater than that in the grain, $D$, so that the diffusion proceeds preferentially through the grain boundary and subsequently into the grain.

For mathematical convenience in derivation of the grain-boundary diffusion equation, the grains are approximated as spheres of radius $a$, and one sphere is chosen at distance $y$ from the surface of the polycrystal, where $y \gg a$.

Equation (1.1) holds for the diffusion in the grain boundary,

$$
D^{\prime} \nabla^{2} C^{\prime}=\frac{\partial C^{\prime}}{\partial t}
$$

where $C^{\prime}$ is the concentration in the grain boundary, and $\boldsymbol{\nabla}^{2}$ denotes the two-dimensional Laplacian operator. The thickness of the grain boundary $\delta$ is considered to be sufficiently small relative to $y$ so that $C^{\prime}$, which is an even function of $x$, can be simply ex-

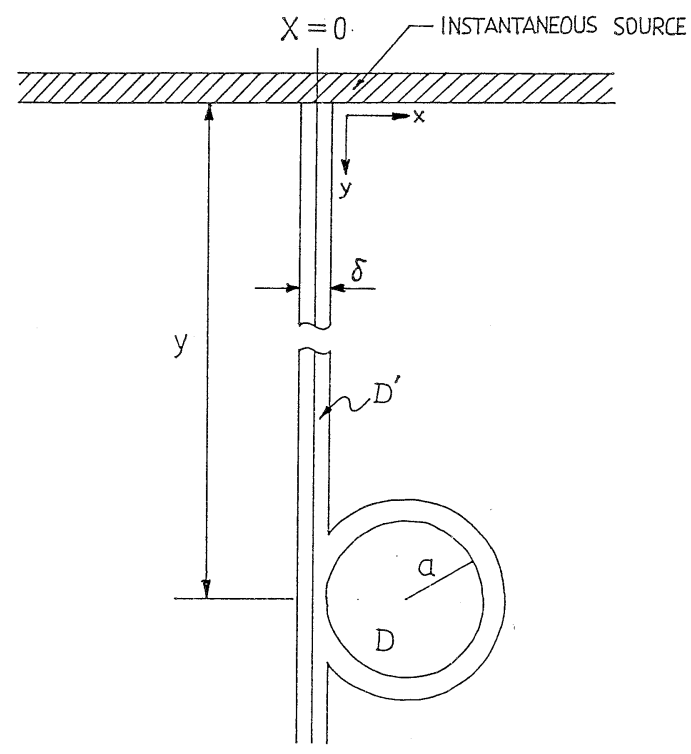

Fig. 1. Schematic diagram of small-grain deep-penetration diffusion from instantaneous source. pressed as Eq. (1.2):

$$
C^{\prime}(x, y, t)=C_{0}{ }^{\prime}(y, t)+\frac{x^{2}}{2} C_{2}{ }^{\prime}(y, t)
$$

Substitution of Eq. (1.2) into Eq. (1.1) gives Eq. (1.3) at the center of the grain boundary, $x=0$.

$$
D^{\prime}\left[\frac{\partial^{2} C_{0}{ }^{\prime}}{\partial y^{2}}+C_{2}{ }^{\prime}\right]=\frac{\partial C_{0}{ }^{\prime}}{\partial t}
$$

On the other hand, at the interface of the grain and the grain boundary, $x=\delta / 2$, the continuity of the material flow is expressed as Eq. (1.4),

$$
\frac{\delta D^{\prime}}{2} C_{2}{ }^{\prime}=\left.D \frac{\partial C}{\partial x}\right|_{x=\delta / 2}
$$

where $C$ is the concentration in the grain. Thus Eqs. (1.3) and (1.4) give Eq. (1.5), which describes the diffusion in the grain boundary.

$$
D^{\prime} \frac{\partial^{2} C_{0}{ }^{\prime}}{\partial y^{2}}+\left.\frac{2 D}{\delta} \frac{\partial C}{\partial x}\right|_{x=\delta / 2}=\frac{\partial C_{0}{ }^{\prime}}{\partial t}
$$

A similar equation has been derived by Whipple ${ }^{3)}$ for description of the boundary condition for the concentration in the bulk.

The second term in the left-hand side of Eq. (1.5) denotes the diffusion flux from the grain boundary into the spherical grain. Therefore, Eq. (1.5) is considered to be a one-dimensional diffusion equation which describes the diffusion along the grain boundary with the total amount of the diffusing substance decreasing due to the flux into the grain. If the flux is known as a function of distance $y$ and time $t$, Eq. (1.5) can be solved for the concentration $C_{0}^{\prime}$ at the center of the grain boundary. Because the diffusion proceeds in the grain boundary much faster than in the bulk, the concentration $C$ in the grain can be calculated under the condition that the concentration outside the grain is kept constant when the diffusion has proceeded to a certain extent.5)

The solution, which is denoted by $C_{\mathrm{s}}$, for the diffusion into a sphere from the grain boundary of constant concentration is given by Eq. (1.6):14)

$$
\frac{C_{\mathrm{s}}-C_{1}}{\Phi-C_{1}}=1+\frac{2 a}{\pi r} \sum_{n=1}^{\infty} \frac{(-1)^{n}}{n} \sin \frac{n \pi r}{a} e^{-D n^{2} \pi^{2} t / a^{2}}
$$

The function $C_{\mathrm{s}}$ satisfies the conditions (1.7a) and (1.7b):

$$
\begin{aligned}
& C_{\mathrm{s}}(r, t=0)=C_{1} \\
& C_{\mathrm{s}}(r=a, t)=\Phi
\end{aligned}
$$

Here $r$ denotes the distance from the center of the sphere, $C_{1}$ the initial concentration in the sphere, and $\Phi$ the constant concentration outside the sphere. Differentiation of $C_{\mathrm{s}}$ with $r$ gives Eq. (1.8), which describes the diffusion flux toward the interior of the bulk at the interface with the grain boundary. 


$$
-\left.D \frac{\partial C_{\mathrm{s}}}{\partial r}\right|_{r=a}=-\left(\Phi-C_{1}\right) \frac{2}{a} \sum_{n=1}^{\infty} e^{-D n^{2} \pi^{2} t / a^{2}}
$$

This flux is equal to the flux at $x=\delta / 2$, which has been described by the second term of the left-hand side in Eq. (1.5). Substitution of Eq. (1.8) in Eq. (1.5) gives Eq. (1.9),

$$
D^{\prime} \frac{\partial^{2} C_{0}{ }^{\prime}}{\partial y^{2}}-\frac{2 D}{\delta}\left(C_{0}{ }^{\prime}-C_{1}\right) \frac{2}{a} \sum_{n=1}^{\infty} e^{-D n^{2} \pi^{2} t / a^{2}}=\frac{\partial C_{0}{ }^{\prime}}{\partial t}
$$

where $\Phi$ in Eq. (1.8) is replaced by $C_{0}^{\prime}$. Equation (1.9) describes the grain-boundary diffusion equation as one-dimensional diffusion with decreasing diffusant which sinks into the grain from the grain boundary.

By introducing a new variable $\varphi$ as

$$
\varphi(y, t)=C_{0}{ }^{\prime}(y, t)-C_{1}
$$

the grain-boundary diffusion equation for the smallgrain deep-penetration condition is rewritten as Eq. (1.11),

$$
D^{\prime} \frac{\partial^{2} \varphi}{\partial y^{2}}+\varphi f(t)=\frac{\partial \varphi}{\partial t}
$$

where $f(t)$ is defined as

$$
f(t)=-\frac{4 D}{\delta a} \sum_{n=1}^{\infty} e^{-D n^{2} \pi^{2} t / a^{2}}
$$

\section{Solution of grain-boundary diffusion equation}

Here Eq. (1.11) for the small-grain deep-penetration diffusion is to be solved for the instantaneous source condition.

To solve Eq. (1.11), we use the familiar method to obtain the solution (2.1) in the product form,

$$
\varphi(y, t)=Y(y) T(t)
$$

where $Y$ and $T$ are functions of $y$ and $t$, respectively. In order for $\varphi$ to satisfy Eq. (1.11), the functions $Y$ and $T$ must satisfy Eqs. (2.2a) and (2.2b), respectively,

$$
\begin{aligned}
& \frac{\mathrm{d}^{2} Y}{\mathrm{~d} y^{2}}=-\mu^{2} Y \\
& \frac{\mathrm{d} T}{\mathrm{~d} t}=\left[-D^{\prime} \mu^{2}+f(t)\right] T
\end{aligned}
$$

where $\mu$ is an arbitrary constant. Solutions of Eqs. $(2.2 \mathrm{a})$ and $(2.2 \mathrm{~b})$ are given respectively by

$$
\begin{aligned}
& Y(y)=C \cos [\mu(y-\lambda)] \\
& T(t)=e^{-D^{\prime} \mu^{2} t+\int \mathrm{d} f(t)}
\end{aligned}
$$

where $C$ and $\lambda$ are arbitrary constants.

Since Eq. (1.11) is a linear equation, the solution $\varphi$ is generally given by a sum of the products $Y(y)$ $T(t)$ for possible values of constants $\mu$ and $\lambda$ as described by Eq. (2.4),

$$
\begin{aligned}
\varphi(y, t)= & \int_{0}^{\infty} \mathrm{d} \mu \int_{-\infty}^{\infty} \mathrm{d} \lambda C(\lambda) \cos [\mu(y-\lambda)] \\
& \times e^{-D^{\prime} \mu^{2} t+(2 a / 3 \delta) \psi(t)}
\end{aligned}
$$

where $C(\lambda)$ is the summation coefficient and $\psi(t)$ is defined as

$$
\psi(t)=\frac{6}{\pi^{2}} \sum_{n=1}^{\infty} \frac{1}{n^{2}} e^{-D n^{2} \pi^{2} t / a^{2}}
$$

Here the definition (1.12) of $f(t)$ is used to derive the expression of $\psi(t)$. The initial condition for the diffusion from an instantaneous source is expressed as

$$
\varphi(y, t=0)=\phi_{0} \theta(-y)
$$

where $\phi_{0}$ is a constant, which gives the initial concentration of the source, and $\theta(z)$ is Heaviside's step function:

$$
\theta(z)= \begin{cases}1, & z>0 \\ 0, & z<0\end{cases}
$$

The summation coefficient $C(\lambda)$ should be determined so that $\varphi$ satisfies Eq. (2.6). The final expression for $C(\lambda)$ is given by Eq. (2.8). (see Appendix A)

$$
C(\lambda)=\frac{1}{\pi} \phi_{0} \theta(-\lambda) e^{-2 a / 3 \delta}
$$

Thus we obtain Eq. (2.9) for the solution $\varphi$ of Eq. (1.11),

$$
\varphi(y, t)=\frac{\phi_{0}}{2} \operatorname{erfc}\left(\frac{y}{2 \sqrt{D^{\prime} t}}\right) e^{(2 a / 3 \delta)[\psi(t)-1]}
$$

where the error function $\operatorname{erfc} z$ is defined by Eq. (2.10):

$$
\operatorname{erfc} z=\frac{2}{\pi} \int_{z}^{\infty} \mathrm{d} \eta e^{-\eta^{2}}
$$

The detailed derivation of Eq. (2.9) is described in Appendix B. The exponential factor on the righthand side of Eq. (2.9) expresses the effect of the diffusion flux into the bulk from the grain boundary. If there is no diffusion into the bulk, Eq. (2.9) is reduced to

$$
\varphi(y, t)=\frac{\phi_{0}}{2} \operatorname{erfc} \frac{y}{2 \sqrt{D^{\prime} t}}
$$

which is the well known solution for the one-dimensional diffusion with an instantaneous source. ${ }^{15)}$ Since the magnitude of the exponential factor is less than one, the value of $\varphi$ given by Eq. (2.9) is always less than that given by Eq. (2.11) because of the existence of the diffusion flux into the grain from the grain boundary.

\section{Average concentration distribution along diffusion direction}

The concentration distribution in the diffusion direction is usually determined by scanning tech- 
nique with EPMA in the case of the small-grain deep-penetration diffusion. The characteristic X-ray intensity for an element to be determined tends to be locally enhanced every time the electron beam crosses a grain boundary so that the obtained concentration distribution does not monotonously decrease but goes up and down. In such a case, in order to make the concentration distribution convenient for the analysis of the grain-boundary diffusion, the EPMA scanning is conducted at a given diffusion distance, perpendicularly to the diffusion direction.5) The average of the obtained inhomogeneous distribution is taken as the concentration at the diffusion distance. Such procedures are repeated at intervals along the diffusion direction to construct the distribution of average concentration against the diffusion distance $y$. This average concentration at $y$ should be equal to the average concentration $\bar{C}$ in a spherical grain located at distance $y$, as given by Eq. (3.1):

$$
\bar{C}=\frac{M_{\mathrm{t}}}{M_{\mathrm{t}=\infty}}\left(C_{0}^{\prime}-C_{1}\right)+C_{1}
$$

Here $M_{\mathrm{t}}$ denotes the total amount of diffusing substance in the sphere at time $t$ as defined by Eq. $(3.2),{ }^{14)}$

$$
M_{\mathrm{t}}=4 \pi \int_{0}^{a} \mathrm{~d} r\left[C(r, t)-C_{1}\right]
$$

where $C(r, t)-C_{1}$ is given by Eq. (1.6), with $C_{\mathrm{s}}$ replaced by $C(r, t)$. By using the explicit form of Eq. (1.6) for the integrand, Eq. (3.3) can be derived. ${ }^{14)}$

$$
\frac{M_{\mathrm{t}}}{M_{\mathrm{t}=\infty}}=1-\psi(t)
$$

Therefore we obtain the Eq. (3.4) for the average concentration $\bar{C}$ from Eqs. (2.9), (3.1) and (3.3),

$$
\begin{aligned}
\bar{C}(y, t)= & \frac{\phi_{0}}{2}[1-\psi(t)] \operatorname{erfc} \frac{y}{2 \sqrt{D^{\prime} t}} \\
& \times e^{(2 \alpha / 3 \delta)[\psi(t)-1]}+C_{1}
\end{aligned}
$$

By using Eq. (3.4), we can calculate the average concentration at given $y$ and $t$, which can be compared with experimental values determined by the scanning technique with EPMA.

In derivation of the present grain-boundary diffusion equation, the grain-boundary path from the surface of the polycrystal to the grain of interest was represented by a linear path, as shown in Fig. 1. This path is shorter than grain-boundary paths in actual polycrystals, which are packed with polyhedra. Therefore, a correction factor for the grain-boundary path is necessary in the derived diffusion equation, Eq. (2.9).

The correction factor can be estimated by regarding Fig. 1 as the cross section of a polycrystal packed with equal-sized polyhedra. The factor is 1.5 for the structure packed with hexagons and $\pi / 2$ for the structure packed with circles, leaving the existence of voids aside. The factor is unity for the checkerboard structure packed with squares. The details of this problem, however, are not discussed here.

\section{Summary}

The grain-boundary diffusion equation, Eq. (1.11), was derived for the small-grain deep-penetration diffusion, and solved for the instantaneous source condition. The obtained solution, Eq. (2.9), consists of two terms, one is the simple diffusion solution for the grain-boundary diffusion only, and the other is the factor which expresses the effect of the flux from the grain boundary toward the interior of the grains.

Equation (3.4), which gives the average concentration at given distance and time, should be useful for determination of the diffusion parameters for the grain boundary and the bulk by tracer technique, and also for the analysis of kinetics in the formation of a grain-boundary phase by preferential diffusion in dielectric ceramics.

\section{[Appendix A]}

Equation (2.8) can be derived from Eq. (2.4) by the following process. By setting $t=0$ in Eq. (2.4), we obtain

$$
\varphi(y, t=0)=e^{2 a / 3 \delta} \int_{0}^{\infty} \mathrm{d} \mu \int_{-\infty}^{\infty} \mathrm{d} \lambda C(\lambda) \cos [\mu(y-\lambda)]
$$

where the formula (A.2) is used:

$$
\sum_{n=1}^{\infty} \frac{1}{n^{2}}=\frac{\pi^{2}}{6}
$$

The initial condition is given by Eq. (2.6):

$$
\varphi(y, t=0)=\phi_{0} \theta(-y)
$$

Equations (A.1) and (2.6) give

$$
\phi_{0} \theta(-y) e^{-2 a / 3 \delta}=\int_{0}^{\infty} \mathrm{d} \mu \int_{-\infty}^{\infty} \mathrm{d} \lambda C(\lambda) \cos [\mu(y-\lambda)]
$$

By Fourier's theorem, Eq. (A.4) holds for any function $g(z)$ :

$$
g(z)=\frac{1}{\pi} \int_{0}^{\infty} \mathrm{d} \mu \int_{-\infty}^{\infty} \mathrm{d} \lambda g(\lambda) \cos [\mu(z-\lambda)]
$$

Comparison of Eqs. (A.3) and (A.4) gives

$$
C(\lambda)=\frac{1}{\pi} \phi_{0} \theta(-\lambda) e^{-2 a / 3 \delta}
$$

[Appendix B]

Equation (2.9) can be derived as follows: Substituting Eq. (2.8) into Eq. (2.4), we obtain

$$
\begin{aligned}
\varphi(y, t)= & \frac{1}{\pi} \phi_{0} e^{(2 a / 3 \delta)[\psi(t)-1]} \int_{0}^{\infty} \mathrm{d} \mu \int_{-\infty}^{\infty} \mathrm{d} \lambda e^{-D^{\prime} \mu^{2} t} \\
& \times \cos [\mu(y-\lambda)]
\end{aligned}
$$

By using the formula

$$
\int_{0}^{\infty} \mathrm{d} z e^{-\beta z^{2}} \cos 2 \alpha z=\frac{1}{2} \sqrt{\frac{\pi}{\beta}} e^{-\alpha^{2} / \beta} \quad(\beta>0)
$$

the integral over $\mu$ can be written as

$$
\int_{0}^{\infty} \mathrm{d} \mu e^{-D^{\prime} \mu^{2} t} \cos [\mu(y-\lambda)]=\frac{1}{2} \sqrt{\frac{\pi}{D^{\prime} t}} e^{-(y-\lambda)^{2} / 4 D^{\prime} t}
$$

$\left(D^{\prime} t>0\right)$ 
Then the function $\varphi$ in Eq. (B.1) is rewritten as

$$
\varphi(y, t)=\frac{\phi_{0}}{2 \sqrt{\pi D^{\prime} t}} e^{(2 a / 3 \delta)[\psi(t)-1]} \int_{-\infty}^{0} \mathrm{~d} \lambda e^{-(y-\lambda)^{2} / 4 D^{\prime} t}(\mathrm{~B} .4)
$$

For the integral of $\lambda$, by replacing the variable as

$$
\eta=\frac{y-\lambda}{2 \sqrt{D^{\prime} t}}
$$

we obtain

$$
\begin{aligned}
\int_{-\infty}^{0} \mathrm{~d} \lambda e^{-(y-\lambda)^{2} / 4 D^{\prime} t} & =-2 \sqrt{D^{\prime} t} \int_{\infty}^{y / 2 \sqrt{D^{\prime} t}} \mathrm{~d} \eta e^{-\eta^{2}} \\
& =\sqrt{\pi D^{\prime} t} \operatorname{erfc} \frac{y}{2 \sqrt{D^{\prime} t}}
\end{aligned}
$$

where the definition (2.10) for the error function is used. Thus we obtain Eq. (2.9):

$$
\varphi(y, t)=\frac{\phi_{0}}{2} \operatorname{erfc}\left(\frac{y}{2 \sqrt{D^{\prime} t}}\right) e^{(2 a / 3 \delta)[\psi(t)-1]}
$$

\section{References}

1) J. C. Fisher, J. Appl. Phys., 22, 74-77 (1951).

2) A. D. Le Claire, Br. J. Appl. Phys., 14, 351-56 (1963).
3) R. T. P. Whipple, Phil. Mag., 45, 1225-36 (1954).

4) T. Suzuoka, Trans. Jpn. Inst. Metals, 2, 25-33 (1961).

5) Y. Oishi and H. Ichimura, J. Chem. Phys., 71, 5134-39 (1979).

6) Y. Oishi, Y. Sakka and K. Ando, J. Nucl. Mater., 96, 23-28 (1981).

7) Y. Oishi and Y. Sakka, J. Nucl. Mater., 97, 44-46 (1981).

8) Y. Sakka, Y. Oishi and K. Ando, Bull. Chem. Soc. Japan, 55, 420-22 (1982).

9) Y. Sakka, Y. Oishi and K. Ando, J. Mater. Sci., 17, 3101-05 (1982).

10) Y. Oishi, K. Ando and Y. Sakka, "Advances in Ceramics, Vol. 7”, Ed. by M. F. Yan and A. H. Heuer, Am. Ceram. Soc., Inc. (1983) pp. 208-19.

11) Y. Sakka, Y. Oishi, K. Ando and Y. Ikeda, J. Am. Ceram. Soc., 76, 1381-83 (1993).

12) Y. Sakka, Y. Oishi, K. Ando and S. Morita, J. Am. Ceram. Soc., 74, 2610-14 (1991).

13) Y. Sakka, Y. Oishi, K. Ando and H. Masuda, J. Am. Ceram. Soc., 72, 2121-25 (1989).

14) J. Crank, "The Mathematics of Diffusion", Clarendon, Oxford (1956) p. 86.

15) J. Crank, ibid., p. 12. 